\title{
Correlation Between Different Stages of Diabetic Nephropathy and Neuropathy in Patients with T2DM: A Cross-Sectional Controlled Study
}

\author{
Lingling Xu (I) $\cdot$ Xiaopu Lin $\cdot$ Meiping Guan $\cdot$ Yingshan Liu
}

Received: August 22, 2018 / Published online: October 9, 2018

(C) The Author(s) 2018

\begin{abstract}
Introduction: Early detection of diabetic peripheral neuropathy (DPN) is critical in patients with type 2 diabetes mellitus (T2DM) due to the lack of targeted therapy for DPN. We have investigated the relationship between different stages of diabetic nephropathy and DPN in an attempt to elucidate whether albuminuria can be used as an early warning signal of DPN progression.

Methods: A total of $217 \mathrm{~T} 2 \mathrm{DM}$ patients who met the inclusion criteria were recruited from the Department of Endocrinology, Nanfang Hospital between January 2016 and June 2016. These patients were placed in groups based on
\end{abstract}

Enhanced digital features To view enhanced digital features for this article go to https://doi.org/10.6084/ m9.figshare.7133672.

Lingling $\mathrm{Xu}$ and Xiaopu Lin contributed equally to this work.

L. Xu (凶)

Department of Endocrinology, Shenzhen Hospital, Southern Medical University, Shenzhen, China e-mail: lucylingl@126.com

X. Lin

Department of Huiqiao Building, Nanfang Hospital, Southern Medical University, Guangzhou, China

M. Guan · Y. Liu

Department of Endocrinology, Nanfang Hospital, Southern Medical University, Guangzhou, China urinary albumin excretion rate (UAER) and estimated glomerular filtration rate. Nerve conduction studies, the Semmes-Weinstein monofilament test (SWMT) and the vibration perception threshold (VPT) test were conducted. Multiple linear regression analysis, multivariate logistic regression and receiveroperating characteristic (ROC) analysis were performed to investigate the relationship between different stages of diabetic nephropathy and DPN in these patients.

Results: Significant differences were observed in the conduction velocity (CV) and amplitude of sensory/motor nerve potential among the T2DM patients at different stages of diabetic nephropathy (all $p<0.05$ ). The UAER and duration of diabetes were found to be independent factors associated with the mean $\mathrm{CV}$ and amplitude of sensory/motor nerve potential (all $p<0.05$ ). A disease duration of $>10$ years $(p=0.025)$ and a higher total cholesterol value $(p=0.024)$ were found to be significantly associated with abnormal SWMT results. A UAER of $>300 \mathrm{mg} / 24 \mathrm{~h}(p=0.007)$ and a diastolic blood pressure of $>100 \mathrm{mmHg}$ ( $p=$ 0.042) were associated with a higher risk for abnormal VPT. A UAER of $>300 \mathrm{mg} / 24 \mathrm{~h}$ $(p<0.001)$ and a disease duration of $>10$ years $(p=0.02)$ were observed to be significantly correlated with DPN. The ROC analysis showed that the optimal cutoff values of UAER and duration as indicators of DPN were $90.5 \mathrm{mg} / 24 \mathrm{~h}$ and 9.5 years, respectively (both $p<0.001$ ). 
Conclusions: The results suggest that diabetic nephropathy is closely associated with the development of DPN in T2DM patients and that UAER and disease duration can be used as warning indicators of DPN progression.

Chinese Clinical Trials Register Number: ChiCTR-ROC-16007701.

Keywords: Diabetic nephropathy; Diabetic peripheral neuropathy; Urinary albumin excretion rate

\section{INTRODUCTION}

Type 2 diabetes mellitus (T2DM) is a group of chronic and systemic metabolic diseases, the prevalence of which has reached epidemic proportions worldwide [1]. Due to microvascular and macrovascular complications, T2DM is associated with increased morbidity and mortality [2]. Nephropathy and neuropathy are two common complications of T2DM that seriously affect patients' quality of life; they are also the leading causes of disability and death in T2DM patients.

The clinical treatment of T2DM patients prioritizes stratification of the risk of various complications at the preclinical stage, during which time the damage is incipient and clinical interventions are likely to be the most effective in preventing progression to more advanced stages. For example, the urinary albumin excretion rate (UAER) at the microalbuminuria stage is considered to be a marker of incipient renal injury in diabetes, and its measurement as a prognostic marker has become firmly entrenched in clinical practice [3]. It has also been noted that once microalbuminuria is present, the average reduction in the creatinine clearance rate in untreated $\mathrm{T} 2 \mathrm{DM}$ patients is approximately $10-12 \mathrm{~mL} / \mathrm{min}$ per year [4]. Thus, determination of the presence or absence of microalbuminuria affects the choice of interventions that may prevent progression to further kidney injury several years before its actual development.

Diabetic peripheral neuropathy (DPN) also appears before the onset of symptoms. This condition is known to be the main initiation factor for foot ulceration and lower extremity amputation [5]. About one-third of patients with DPN develop pain, which has severe consequences on the quality of life $[5,6]$. Despite DPN being the most common complication of diabetes, many of its underlying pathophysiological mechanisms are unknown, and there is a lack of targeted therapy. Consequently, early diagnosis and treatment initiation are critical, with identification at an early stage likely providing an opportunity for effective intervention $[7,8]$.

Thus, objective tests and prognostic markers that can be used to identify DPN during its early stage are critical. Nerve conduction studies (NCSs) are considered to be the most accurate and reliable methods for detecting DPN [9-11] and ideally should be performed routinely in all patients with T2DM. However, NCSs are extremely costly in terms of financial and human resources, thus limiting their routine use.

Screening for albuminuria is a routine clinical examination and commonly carried out in T2DM patients at admission to the hospital. The primary aim of this study was to evaluate the possible close correlation between different stages of diabetic nephropathy and the occurrence of DPN. Additionally, we attempted to elucidate whether albuminuria can be used as a warning signal of DPN progression, with the aim to select T2DM patients at high risk of DPN for early screening and intervention.

\section{METHODS}

\section{Study Design}

This was a cross-sectional, open-label, controlled clinical study, with the aim to investigate the association between different stages of diabetic nephropathy and neuropathy. All procedures performed in the study are in accordance with the Ethics Committee of Nanfang Hospital, Southern Medical University and with the 1964 Helsinki declaration and its later amendments or comparable ethical standards. Informed consent was obtained from all individual participants included in the study. The 
Chinese Clinical Trials Registration Number of the present study is ChiCTR-ROC-16007701.

\section{Participants}

A total of 259 patients with T2DM were initally recruited from the Department of Endocrinology of Nanfang Hospital between January 2016 and June 2016, of whom 42 patients were excluded according to the exclusion criteria. The mean age of the 217 patients who were enrolled in the study (136 males, 81 females) was $59.26 \pm 11.12$ years, and the mean duration of diabetes at enrollment was $9.38 \pm 6.05$ years. All patients underwent tests to screen for diabetic nephropathy and neuropathy.

The exclusion criteria for the patients were: kidney transplantation, nondiabetic nephropathy kidney diseases, cervical vertebra and lumbar intervertebral disc herniation, unstable medical state (acute cerebrovascular events, acute coronary events, transaminase level more than twofold higher than normal, serum potassium $>5.5 \mathrm{mmol} / \mathrm{L}$ and acute infectious diseases), malignant tumor, limb trauma, chronic rheumatic disease, thyroid function abnormalities, alcohol abuse, intake of neurotoxic medication, vitamin B12 deficiency and pregnancy.

Patients were classified into four groups based on UAER and estimated glomerular filtration rate (eGFR): normoalbuminuria group (NG; UAER $<30 \mathrm{mg} / 24 \mathrm{~h}, n=99$ ); microalbuminuria group (MG; UAER $\geq 30 \mathrm{mg} / 24 \mathrm{~h}$ and $<300 \mathrm{mg} / 24 \mathrm{~h}, \quad n=45)$; proteinuria group (PG; UAER $\geq 300 \mathrm{mg} / 24 \mathrm{~h}$ and eGFR [according to Chronic Kidney Disease Epidemiology Collaboration] $\geq 60 \mathrm{~mL} / \mathrm{min} / 1.73 \mathrm{~m}^{2}$, $n=38$; renal insufficiency group (RIG; UAER $\geq 300 \mathrm{mg} / 24 \mathrm{~h}$ and eGFR $<60 \mathrm{~mL} / \mathrm{min} /$ $\left.1.73 \mathrm{~m}^{2}, n=35\right)$ [12].

\section{Measurements}

Each patient's medical history was retrieved from hospital records and the information supplemented with measurements and tests performed on the first day after admission. Body height and weight were measured with the patient wearing light clothes and in bare feet. All urine and blood samples were collected after an overnight fast (minimum duration $8 \mathrm{~h}$ ). Albuminuria was measured in a 24-h urine collection.

The levels of serum uric acid (SUA), lowdensity lipoprotein (LDL), high-density lipoprotein (HDL), total cholesterol (TC), triglycerides (TG) and fasting plasma glucose were measured using a biochemical automatic analyzer (model AU5431; Beckman Coulter, Brea, CA, USA), as was the concentration of urinary creatinine (model AU2700; Beckman Coulter). Urinary albumin was measured on a Siemens Dade Behring BN II Nephelometer (Siemens AG, Munich, Germany). Glycated hemoglobin (HbA1c) was estimated by highperformance liquid chromatography (Tosoh Corp., Tokyo, Japan). The urinary albumin to creatinine ratio (UACR) was estimated by dividing the urinary albumin concentration by the urinary creatinine concentration.

The Nicolet Viking Quest electromyograph system (VIASYS Healthcare, Conshohocken, PA, USA) was used for electrophysiological assessments by the same electrophysiologist. Sensory (S) nerve studies were conducted using the ulnar, median, and sural nerves, and motor (M) nerve studies were conducted using the ulnar, median, peroneal and posterior tibial nerves. The conduction velocity $(\mathrm{CV})$, sensory nerve action potential (SNAP) amplitude and compound muscle action potential (CMAP) amplitude were measured and recorded. The mean value of the sensory $\mathrm{CV}$ was calculated as: $\mathrm{CV}_{\text {sensory nerve }}=\left(\mathrm{CV}_{\text {ulnar nerve } \mathrm{S}}+\mathrm{CV}_{\text {median nerve }} \mathrm{S}\right.$ $+\mathrm{CV}_{\text {sural nerve } \mathrm{s}} / 3$. The same method was used for the mean of motor nerve CV, SNAP amplitude and CMAP amplitude, respectively.

The quantitative sensory tests, including the Semmes-Weinstein monofilament test (SWMT) and the vibration perception threshold (VPT) test, were also performed to evaluate neuropathy in this study. The SWMT method used is described in our previous study [13]. SWMT results were considered to be abnormal when at least two of the five testing sites were insensate on both feet [14]. The VPT was measured in a standardized manner using a biothesiometer 
(Bio-Medical Instrument Co., Newbury, OH, USA) [15]. The higher mean VPT value in either limb was used for analysis. VPT test values were considered to be abnormal when the value exceeded $25 \mathrm{mV}$ [16].

In this study, the presence of DPN was defined when there was an abnormal result for the electrophysiological assessment ( $\mathrm{CV}$ or amplitude) in at least two different nerves, combined with abnormal VPT or SWMT results.

\section{Statistical Analysis}

For continuous variables, results were presented as the mean \pm standard deviation if normally distributed and the median and interquartile range if non-normally distributed. The one-way analysis of variance test (normally distributed data) and the Kruskal-Wallis test (nonparametric data) were employed to examine differences among groups. The categorical data were analyzed using Pearson's $\chi^{2}$ test. To evaluate the correlation between the clinical variables and DPN, we performed multiple linear regression analysis (the mean of CV and amplitude as the dependent variables) and multivariate logistic regression (the results of VPT, SWMT, and DPN as the dependent variables). Receiver-operating characteristic (ROC) analysis was performed to identify the optimal cutoff values for duration and UAER as indicators of DPN. A two-sided $p$ value of $<0.05$ was assumed to be statistically significant while a $p$ value of $<0.001$ was taken to be highly significant. All statistical analyses were performed using SPSS statistics software (version 19.0, 2010; IBM Corp., Armonk, NY, USA).

\section{RESULTS}

\section{Clinical Characteristics of Enrolled Patients}

The baseline characteristics of the patients are shown in Table 1. Duration, systolic blood pressure (SBP), UACR and UAER were statistically significantly different (all $p<0.05$ ) between each patient group (NG, MG, PG and
RIG), but there were no notable inter-group differences for the other measures.

\section{NCS Variables at Different Stages of Diabetic Nephropathy}

Statistically significant differences were seen in the CV and amplitude of the sensory/motor nerve action potential between each group of T2DM patients at different stages of diabetic nephropathy (Table 2).

\section{Correlation Between Clinical Variables and NCS}

Due to the high inter-correlation between UAER and UACR ( $p<0.001$, data not shown), the UACR data were excluded from the multiple linear regression in order to avoid multicollinearity. Correlation analysis was performed between the clinical variables and the mean $\mathrm{CV} /$ amplitude levels of the sensory/motor nerves. The results revealed that the duration of diabetes, SBP, HbA1c, TC, HDL and UAER were associated to the means of the NCS variables (data not shown).

We then performed multiple linear regression analysis using the mean $\mathrm{CV}$ and amplitude levels of the sensory/motor nerves tested as dependent variables, respectively, and the above selected clinical variables as independent variables. Multiple linear regression revealed that UAER and duration were independent factors associated with the mean sensory nerve $\mathrm{CV}$. Duration, SBP, and UAER were independent factors associated with the mean sensory nerve amplitude. UAER, duration, TC, and HbA1c were independent factors associated with the mean motor nerve $\mathrm{CV}$, and UAER, duration, and SBP were independent factors associated with the mean motor nerve amplitude (Table 3).

\section{Risk Factors for abnormal SWMT and VPT results in T2DM Patients}

We transformed the continuous variables into grade variables according to pre-determined intervals for logistic regression analysis as follows: age ( $\leq 60$ years, $>60$ years), diabetes 
Table 1 Clinical characteristics of patients with type 2 diabetes mellitus at different stages of diabetic nephropathy

\begin{tabular}{|c|c|c|c|c|c|}
\hline \multirow{2}{*}{$\begin{array}{l}\text { Patient baseline } \\
\text { characteristics }\end{array}$} & \multicolumn{4}{|l|}{ Patient groups $^{\mathrm{a}}$} & \multirow[t]{2}{*}{$p$ value } \\
\hline & NG & MG & PG & RIG & \\
\hline Number (males/females) & $99(68 / 31)$ & $45(27 / 18)$ & $38(20 / 18)$ & $35(21 / 14)$ & $>0.05$ \\
\hline Age (years) & $57.81 \pm 11.24$ & $58.98 \pm 11.64$ & $61.15 \pm 9.87$ & $64.09 \pm 9.91$ & $>0.05$ \\
\hline Duration (years) & $7.85 \pm 6.70$ & $10.27 \pm 7.85$ & $10.44 \pm 6.30$ & $13.41 \pm 5.96$ & $<0.05^{*}$ \\
\hline Smoking (\%) & 34.69 & 46.67 & 22.22 & 27.27 & $>0.05$ \\
\hline BMI $\left(\mathrm{kg} / \mathrm{m}^{2}\right)$ & $24.57 \pm 5.23$ & $25.23 \pm 3.44$ & $26.04 \pm 3.57$ & $24.52 \pm 2.21$ & $>0.05$ \\
\hline SBP $(\mathrm{mmHg})$ & $134.57 \pm 18.36$ & $138.83 \pm 18.90$ & $148.70 \pm 22.41$ & $160.64 \pm 20.68$ & $<0.001^{* *}$ \\
\hline $\mathrm{DBP}(\mathrm{mmHg})$ & $79.45 \pm 10.93$ & $80.17 \pm 11.39$ & $82.85 \pm 10.52$ & $81.77 \pm 14.02$ & $>0.05$ \\
\hline SUA $(\mathrm{mmol} / \mathrm{L})$ & $373.17 \pm 98.98$ & $379.21 \pm 93.94$ & $400.44 \pm 103.87$ & $434.32 \pm 93.47$ & $>0.05$ \\
\hline $\mathrm{LDL}(\mathrm{mmol} / \mathrm{L})$ & $3.31 \pm 1.05$ & $3.14 \pm 1.13$ & $3.23 \pm 0.88$ & $3.16 \pm 1.19$ & $>0.05$ \\
\hline $\mathrm{HDL}(\mathrm{mmol} / \mathrm{L})$ & $1.03 \pm 0.23$ & $0.99 \pm 0.27$ & $1.00 \pm 0.30$ & $0.90 \pm 0.18$ & $>0.05$ \\
\hline $\mathrm{TC}(\mathrm{mmol} / \mathrm{L})$ & $5.02 \pm 1.10$ & $4.97 \pm 1.40$ & $5.09 \pm 1.27$ & $5.03 \pm 1.58$ & $>0.05$ \\
\hline $\mathrm{TG}(\mathrm{mmol} / \mathrm{L})$ & $1.91 \pm 1.29$ & $2.24 \pm 1.60$ & $2.56 \pm 2.81$ & $2.29 \pm 1.28$ & $>0.05$ \\
\hline $\mathrm{FPG}(\mathrm{mmol} / \mathrm{L})$ & $8.21 \pm 3.84$ & $8.81 \pm 3.33$ & $8.85 \pm 3.07$ & $8.31 \pm 6.77$ & $>0.05$ \\
\hline HbAlc (\%) & $\begin{array}{l}7.80 \\
\quad(6.90-9.43)\end{array}$ & $\begin{array}{l}8.90 \\
\quad(7.25-10.78)\end{array}$ & $9.00(7.45-9.95)$ & $7.75(6.53-8.58)$ & $>0.05$ \\
\hline UACR $(\mathrm{mg} / \mathrm{g})$ & $\begin{array}{l}6.19 \\
\quad(3.98-10.61)\end{array}$ & $\begin{array}{l}52.17 \\
\quad(22.99-98.14)\end{array}$ & $\begin{array}{l}580.90 \\
\quad(294.43-1069.85)\end{array}$ & $\begin{array}{l}1506.18 \\
\quad(761.27-3864.71)\end{array}$ & $<0.001^{* *}$ \\
\hline UAER (mg/24 h) & $\begin{array}{l}7.00 \\
\quad(5.00-12.25)\end{array}$ & $\begin{array}{l}73.00 \\
\quad(39.25-122.25)\end{array}$ & $\begin{array}{l}806.00 \\
\quad(398.50-995.50)\end{array}$ & $\begin{array}{l}1550.00 \\
\quad(565.00-2844.00)\end{array}$ & $<0.001^{* *}$ \\
\hline
\end{tabular}

Values are expressed as the mean \pm standard deviation (SD) for normally distributed data and as the median with the interquartile range (IQR) in parenthesis for non-normally distributed data, unless indicated otherwise. Differences between each patient group were analyzed by one-way analysis of variance (ANOVA) for normally distributed values and by the Kruskal-Wallis test for nonparametric values. Pearson's $\chi^{2}$ test was employed to analyze categorical data

$B M I$ Body max index, SBP systolic blood pressure, DBP diastolic blood pressure, $S U A$ serum urine acid, $L D L$ low-density lipoprotein, $H D L$ high-density lipoprotein, $T C$ total cholesterol, $T G$ triglycerides, $F P G$ fasting plasma glucose, $H b A 1 c$ glycated hemoglobin, $U A C R$ urinary albumin to creatinine ratio, UAER urinary albumin excretion rate ${ }^{*} p<0.05,{ }^{* *} p<0.001$

a Patients were classified into four groups based on UAER and estimated glomerular filtration rate (eGFR): normoalbuminuria group (NG; microalbuminuria group (MG; proteinuria group (PG); renal insufficiency group (RIG). For more detail see section Participants

duration $(\leq 10,>10$ years $)$, SBP $(\leq 140,140$ $160,>160 \mathrm{mmHg})$, diastolic BP (DBP; $\leq 90$, 90-100, > $100 \mathrm{mmHg}$ ), body mass index (BMI; $\left.\leq 24,24-28,>28 \mathrm{~kg} / \mathrm{m}^{2}\right), \quad H b A 1 \mathrm{c} \quad(\leq 6 \%$, $>6 \%)$, SUA $(\leq 357,>357 \mu \mathrm{mol} / \mathrm{L}), \mathrm{LDL}(\leq 3.3$, $>3.3 \mathrm{mmol} / \mathrm{L})$, HDL $(\leq 1.03,>1.03 \mathrm{mmol} / \mathrm{L})$, TC $(\leq 5.2,>5.2 \mathrm{mmol} / \mathrm{L}), \quad$ TG $(\leq 1.7,>1.7$ $\mathrm{mmol} / \mathrm{L})$ and UAER $(\leq 30,30-300,>300 \mathrm{mg} /$ $24 \mathrm{~h})$.

Multivariate logistic regression analysis was used to assess the SWMT and VPT results as dependent variables, respectively, and age, sex, BMI, duration of diabetes, smoking history, SBP, DBP, HbA1c, SUA, LDL, HDL, TC, TG and UAER 
Table 2 Nerve conduction variables in patients with type 2 diabetes mellitus at different stages of diabetic nephropathy

\section{Nerve conduction variables}

$\mathrm{DPN} /$ total $n$

Ulnar nerve $S$

$$
\mathrm{CV}(\mathrm{m} / \mathrm{s})
$$

Amp (mV)

Median nerve S

$$
\mathrm{CV}(\mathrm{m} / \mathrm{s})
$$$$
\text { Amp (mV) }
$$

Sural nerve $S$

$$
\begin{aligned}
& \mathrm{CV}(\mathrm{m} / \mathrm{s}) \\
& \text { Amp }(\mathrm{mV})
\end{aligned}
$$

(52.00-61.00)

41.70

(26.30-55.00)

50.00

(44.00-54.00)

17.60

(12.00-28.80)

Ulnar nerve $\mathrm{M}$

$$
\begin{aligned}
& \mathrm{CV}(\mathrm{m} / \mathrm{s}) \\
& \text { Amp }(\mathrm{mV})
\end{aligned}
$$

$$
\begin{aligned}
& 55.56 \pm 6.65 \\
& 12.21 \pm 3.37
\end{aligned}
$$

$$
\begin{aligned}
& 54.22 \pm 9.35 \\
& 12.07 \pm 5.65
\end{aligned}
$$

$$
\text { (43.00-53.00) }
$$$$
45.00
$$$$
\text { (15.00-51.00) }
$$$$
52.00
$$$$
\text { (38.00-57.00) }
$$$$
39.00
$$$$
<0.001^{* *}
$$

(10.50-33.60)

$$
\text { (9.00-49.00) }
$$$$
7.50(1.40-18.20)<0.001^{* *}
$$

$p$ value

Median nerve $\mathrm{M}$

$$
\begin{aligned}
& \mathrm{CV}(\mathrm{m} / \mathrm{s}) \\
& \text { Amp }(\mathrm{mV})
\end{aligned}
$$$$
54.08 \pm 3.86
$$$$
52.49 \pm 4.69
$$$$
11.33 \pm 3.50
$$$$
9.94 \pm 3.27
$$

$$
\begin{aligned}
& 52.11 \pm 4.69 \\
& 10.31 \pm 3.35
\end{aligned}
$$

$$
47.14 \pm 4.82
$$$$
<0.001^{* *}
$$

$$
\begin{aligned}
& 44.61 \pm 4.78 \\
& 6.00(4.20-8.60)
\end{aligned}
$$$$
42.02 \pm 6.75
$$$$
5.20(2.45-7.40)
$$

$40.11 \pm 7.94$

$4.00(1.40-7.00)$

$7.45 \pm 2.91$

$<0.001^{* *}$

$0.006^{*}$ $0.002^{*}$

$$
\begin{aligned}
& 46.26 \pm 6.83 \\
& 11.90
\end{aligned}
$$

(8.90-15.80)

$$
\begin{aligned}
& 43.53 \pm 7.34 \\
& 11.60
\end{aligned}
$$

(6.15-15.70)
$41.41 \pm 6.27$

10.10

(6.10-13.10)

Data are presented as the mean $\pm S D$ or the median with the IQR in parenthesis, unless indicated otherwise. Differences among the groups were analyzed by ANOVA or Kruskal-Wallis test.

$D P N$ Diabetic peripheral neuropathy, $S$ sensory nerve study, $M$ motor nerve study, $C V$ conduction velocity, $A m p$ amplitude ${ }^{*} p<0.05,{ }^{* *} p<0.001$ 
Table 3 Multiple linear regression analysis of nerve conduction study results and clinical variables

\begin{tabular}{llll}
\hline Dependent variables & Independent variables & $\boldsymbol{\beta}(\mathbf{9 5} \% \mathbf{C l})$ & $\boldsymbol{p}$ value \\
\hline Sensory nerve CV & UAER & $-0.004(-0.005,-0.003)$ & $<0.001^{* *}$ \\
Sensory nerve Amp & Duration & $-0.699(-0.832,-0.560)$ & $<0.05^{*}$ \\
& Duration & $-1.000(-1.214,-0.590)$ & $<0.001^{* *}$ \\
SBP & $-0.120(-0.232,-0.015)$ & $<0.05^{*}$ \\
Motor nerve CV & UAER & $-0.002(-0.005,-0.001)$ & $<0.05^{*}$ \\
& UAER & $-0.002(-0.003,-0.001)$ & $<0.001^{* *}$ \\
Duration & $-0.245(-0.321,-0.174)$ & $<0.001^{* *}$ \\
TC & $-0.835(-0.946,-0.696)$ & $<0.05^{*}$ \\
Motor nerve Amp & HbA1c & $-0.308(-0.436,-0.281)$ & $<0.05^{*}$ \\
& UAER & $-0.001(-0.002,-0.000)$ & $<0.05^{*}$ \\
& Duration & $-0.099(-0.155,-0.061)$ & $<0.001^{* *}$ \\
\hline SBP & $-0.027(-0.034,-0.013)$ & $<0.05^{*}$ \\
\hline
\end{tabular}

95\% CI 95\% Confidential interval

${ }^{*} p<0.05,{ }^{* *} p<0.001$

as independent variables. The results are shown in Table 4.

T2DM duration of $>10$ years (odds ratio [OR] 3.12, 95\% confidence interval [CI] $1.15-8.45 ; p=0.025)$ was significantly associated with abnormal SWMT results. A TC level higher than the normal level increased the risk for an abnormal SWMT result by fourfold (OR $4.53,95 \%$ CI $1.22-16.89 ; p=0.024)$. A UAER of $>300 \mathrm{mg} / 24 \mathrm{~h}$ was found to be positively associated with an abnormal VPT test results (OR 7.65, 95\% CI 1.73-33.85; $p=0.007$ ). Similarly, a DBP of $>100 \mathrm{mmHg}$ was associated with a higher risk for an abnormal VPT test result (OR 7.89, 95\% CI 1.08-57.96, $p=0.042$ ).

\section{Risk Factors for DPN in T2DM Patients}

To identify potential risk factors for DPN, we performed multivariate logistic regression analysis with clinical variables as independent variables, including age, sex, BMI, duration of diabetes, smoking history, SBP, DBP, HbA1c, SUA, LDL, HDL, TC, TG and UAER, in the form of qualitative variables. A UAER of $>300 \mathrm{mg} /$ $24 \mathrm{~h}$ was found to be significantly associated with DPN (OR 7.06, 95\% CI 2.76-18.07; $p<0.001$, and a duration of diabetes of $>10$ years (OR 2.82, 95\% CI 1.18-6.74; $p=0.02$ ) was found to be associated with a higher risk for DPN (Table 4). As UAER and diabetes duration were considered to be independent risk factors of DPN, a ROC analysis was performed to further explore the cutoff point of UAER level and diabetes duration for predicting DPN.

The ROC analysis showed that the optimal cutoff point of UAER to indicate DPN was $90.5 \mathrm{mg} / 24 \mathrm{~h}$ (area under the curve [AUC] 0.706; 95\% CI 0.624-0.789; sensitivity 57.7\%; specificity $84.5 \%$, accuracy $65.9 \%$; precision $59.6 \%$; recall $70.1 \%$; $p<0.001)$. The optimal cutoff point of T2DM duration to indicate DPN was 9.5 years (AUC 0.691; 95\% CI 0.612-0.770; sensitivity $67.9 \%$; specificity, $64.5 \%$, accuracy $77.4 \%$; precision $73.8 \%$; recall $60.8 \%$; $p<0.001$ ) (Fig. 1).

\section{DISCUSSION}

In our study group of patients with T2DM the clinical symptoms of complications associated 
Table 4 Multivariate logistic regression analysis of risk factors for abnormal Semmes-Weinstein monofilament test/ vibration perception threshold test results and diabetic peripheral neuropathy

\begin{tabular}{|c|c|c|c|}
\hline Dependent variables & Independent variables & Odd ratio $(95 \% \mathrm{Cl})$ & $p$ value \\
\hline \multirow[t]{6}{*}{ SWMT } & Duration (years) & & \\
\hline & $\leq 10$ & 1 (Reference) & \\
\hline & $>10$ & $3.12(1.15-8.45)$ & $0.025^{*}$ \\
\hline & $\mathrm{TC}(\mathrm{mmol} / \mathrm{L})$ & & \\
\hline & $\leq 5.2$ & 1 (Reference) & \\
\hline & $>5.2$ & $4.53(1.22-16.89)$ & $0.024^{*}$ \\
\hline \multirow[t]{8}{*}{ VPT } & UAER (mg/24 h) & & \\
\hline & $\leq 30$ & 1 (Reference) & \\
\hline & $30-300$ & $1.15(0.25-5.32)$ & 0.86 \\
\hline & $>300$ & $7.65(1.73-33.85)$ & $0.007^{*}$ \\
\hline & $\mathrm{DBP}(\mathrm{mmHg})$ & & \\
\hline & $\leq 90$ & 1 (Reference) & \\
\hline & $90-100$ & $2.15(0.39-11.94)$ & 0.381 \\
\hline & $>100$ & $7.89(1.08-57.96)$ & $0.042^{*}$ \\
\hline \multirow[t]{7}{*}{ DPN } & UAER (mg/24h) & & \\
\hline & $\leq 30$ & 1 (Reference) & \\
\hline & $30-300$ & $1.55(0.68-3.52)$ & 0.30 \\
\hline & $>300$ & $7.06(2.76-18.07)$ & $<0.001^{* *}$ \\
\hline & Duration (years) & & \\
\hline & $\leq 10$ & 1 (Reference) & \\
\hline & $>10$ & $2.82(1.18-6.74)$ & $0.02^{*}$ \\
\hline
\end{tabular}

SWMT Semmes-Weinstein monofilament test, VPT vibration perception threshold

${ }^{*} p<0.05,{ }^{* *} p<0.001$

with T2DM appeared later than the pathological changes. Estimates of microalbuminuria and the results of funduscopic examinations are known to serve as objective indicators of incipient nephropathy and retinopathy in diabetes. However, evidence for the validity of a comparable objective criterion for the risk for early diabetic neuropathy is lacking.

To date, NCSs are considered to be a consistent indicator or the gold standard of neuropathy [17]. Nevertheless, rigorous control of all aspects of each NCS is critical, and each complete study may require up to $45 \mathrm{~min}$. Consequently, such studies are expensive in terms of manpower, time and cost, and it is therefore not cost-effective to perform NCSs on all T2DM patients. Thus, it is imperative to identify a fairly straightforward warning indicator of diabetic neuropathy to screen high-risk patients and facilitate an early diagnosis and treatment of neuropathy in a timely and efficient manner.

In the study reported here, we performed nephropathy staging in T2DM patients based 


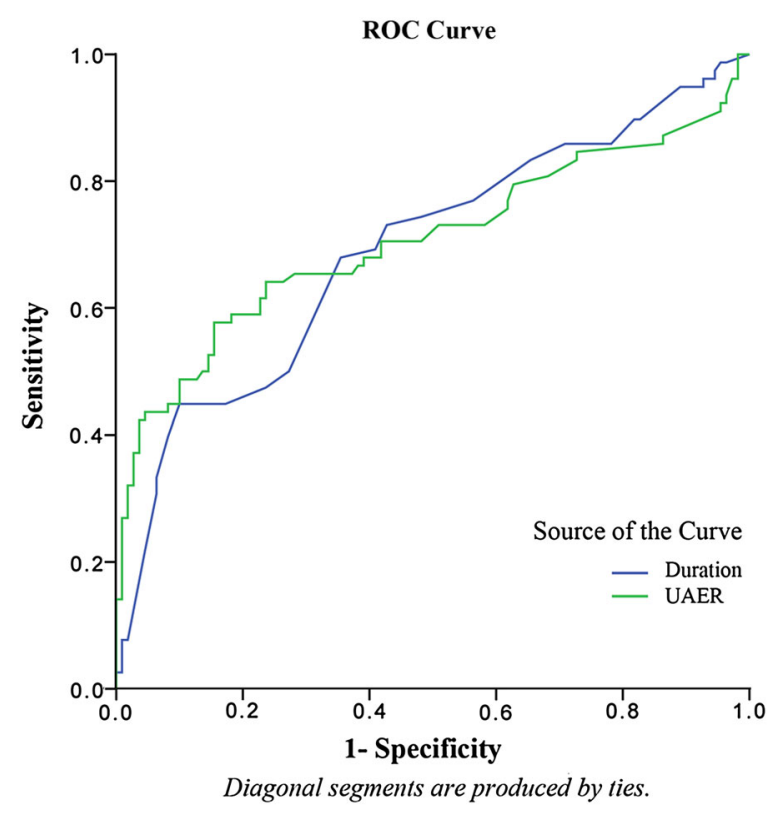

Fig. 1 Receiver-operating characteristic $(R O C)$ analysis of urinary albumin excretion rate (UAER) and duration to predict diabetic nephropathy in patients with type 2 diabetes mellitus (UAER: area under the curve [AUC] 0.706, 95\% confidence interval [CI] 0.624-0.789, sensitivity $57.7 \%$, specificity $84.5 \%$, accuracy $65.9 \%$, precision 59.6\%, recall 70.1\%; $p<0.001$ ) (duration: AUC 0.691, 95\% CI $0.612-0.770$, sensitivity $67.9 \%$, specificity $64.5 \%$, accuracy $77.4 \%$, precision $73.8 \%$, recall $60.8 \%$; $p<0.001$ )

on UAER and eGFR values and found that nerve conduction (including amplitude and CV) significantly decreased with the progression of diabetic nephropathy. In addition, we found that UAER and disease duration were independent risk factors for diabetic neuropathy even when the CV and amplitude of the sensory/motor nerves were used as dependent variables. Furthermore, UAER and disease duration were also independent risk factors leading to abnormal SWMT and VPT test results. These findings suggest that UAER and disease duration can likely be used as warning indicators for the occurrence of DPN.

The definition of DPN used in our study was based on standard published consensus guidelines [18] such that DPN can be confirmed in the presence of abnormal nerve conduction and a symptom(s) or a sign(s) of neuropathy. We defined DPN as the presence of abnormal electrophysiological assessment (CV or amplitude) in at least two different nerves combined with an abnormal VPT test or SWMT result.

SWMT is a simple, accurate, and practical tool for diabetic neuropathy screening [14, 19-21]. A 4-year follow-up study [22] found that SWMT is appropriate as a simple diabetic neuropathy screening instrument generalizable to the clinical setting. VPT tests may also provide vital clinical information on the function of large nerve fibers in diabetic patients, and one study [23] showed that VPT was a sensitive predictor of clinical neuropathy.

In our study, multivariate logistic regression analysis demonstrated that UAER and disease duration were independent risk factors for DPN. The ROC analysis revealed that the risk of DPN will increase significantly in T2DM patients when the UAER is $>90.5 \mathrm{mg} / 24 \mathrm{~h}$ or the duration of diabetes is $>9.5$ years.

Albuminuria has been proposed to be an independent predictor of increasing levels of vascular risk factors and microvascular and macrovascular diseases in T2DM patients [24]. More specifically, albuminuria has been confirmed to be a marker of incipient renal injury in diabetes [3]. In Chinese diabetic patients, a UACR of $>5.6 \mathrm{mg} / \mathrm{mmol}$ predicts progression to renal failure and early death [25]. Several studies have reported a correlation between albuminuria and diabetic retinopathy in T2DM patients. One recent study showed a strong correlation between the severity of diabetic nephropathy and retinopathy and that renal injury precedes retinal damage in T2DM patients [26]. Albuminuria is also considered to be a marker of cardiovascular diseases and a factor which increases the risk of morbidity and mortality $[27,28]$.

However, the number of published studies with the specific aim of investigating the correlation between albuminuria and DPN are very limited, although the results of some studies may indirectly reflect this association. Savage et al. [29] showed that an increasing UAER in a large non-insulin-dependent DM (NIDDM) population was associated with an increased prevalence of diabetic neuropathy, retinopathy and cardiovascular disease, which suggested that UAER may be more than an indicator of renal disease in NIDDM patients. In a study 
conducted in China, Hu et al. [30] showed that high serum cystatin $\mathrm{C}$ level was closely associated with DPN and that this molecule may be a potential biomarker for DPN in T2DM patients. In their review, Callaghan et al. [31] proposed that neuropathy can be particularly severe when diabetic chronic kidney disease occurs. Also, with respect to the association between albuminuria and diabetic autonomic neuropathy, Chuang et al. [32] showed that albuminuria was an independent risk factor for erectile dysfunction in men with T2DM. These results are consistent with the findings of our study in showing a close correlation between albuminuria and the occurrence of DPN. Moreover, a very recent retrospective study [33] also found an association between elevated UACR and decreased eGFR and DPN. Although the results of our study are similar to those reported earlier, the overall design of our study was different, and our analysis of risk factors leading to abnormal SWMT and VPT test results may add to the reliability of results.

In our study, due to high inter-correlation between the UAER and UACR and taking into consideration that the patients were hospitalized and that UAER assessment may improve the accuracy of the results, we excluded UACR from further statistical analysis. However, we consider that, for outpatients, determining the UACR may be more easily performed; our results (data not shown) demonstrated that UACR could also be used as a warning indicator of DPN progress. Therefore, based on the results of our study, we suggest that the UAER can be used as a predictor of diabetic neuropathy as well as for a marker of diabetic nephropathy. For T2DM patients with a UAER of $>90.5 \mathrm{mg} /$ $24 \mathrm{~h}$ or disease duration of $>9.5$ years, comprehensive neurological screening and timely treatment should be carried out.

As a cross-sectional study, the results suggest that UAER and diabetes duration in our T2DM patients were associated with increased risk of DPN progression. Prospective studies with larger sample size and long-term follow-up might provide a more convincible conclusion.

\section{CONCLUSIONS}

In our patients with T2DM, diabetic nephropathy was closely associated with DPN. The UAER and disease duration were found to be indicators that warned of DPN progress.

\section{ACKNOWLEDGEMENTS}

We thank the participants of the study.

Funding. This study was supported by grant from the National Natural Science Foundation of China (No. 81500656) and Natural Science Fund of Guangdong Province (No. 2016A030313521). Article processing charges were funded by the authors.

Authorship. All named authors meet the International Committee of Medical Journal Editors (ICMJE) criteria for authorship for this article, take responsibility for the integrity of the work as a whole, and have given their approval for this version to be published.

Disclosures. Lingling $\mathrm{Xu}$, Xiaopu Lin, Meiping Guan, and Yingshan Liu have nothing to disclose.

Compliance with Ethics Guidelines. All procedures performed in studies involving human participants were in accordance with the ethical standards of the institutional and/or national research committee and with the 1964 Helsinki declaration and its later amendments or comparable ethical standards. Informed consent was obtained from all individual participants included in the study.

Data Availability. The datasets generated during and/or analyzed during the current study are available from the corresponding author on reasonable request.

Open Access. This article is distributed under the terms of the Creative Commons 
Attribution-NonCommercial 4.0 International License (http://creativecommons.org/licenses/ by-nc/4.0/), which permits any noncommercial use, distribution, and reproduction in any medium, provided you give appropriate credit to the original author(s) and the source, provide a link to the Creative Commons license, and indicate if changes were made.

\section{REFERENCES}

1. Haw JS, Galaviz KI, Straus AN, et al. Long-term sustainability of diabetes prevention approaches: a systematic review and meta-analysis of randomized clinical trials. JAMA Intern Med. 2017;177(12):1808-17.

2. Zheng Y, Ley SH, Hu FB. Global aetiology and epidemiology of type 2 diabetes mellitus and its complications. Nat Rev Endocrinol. 2018;14(2):88-98.

3. Williams ME. Diabetic nephropathy: the proteinuria hypothesis. Am J Nephrol. 2005;25(2):77-94.

4. Remuzzi G, Schieppati A, Ruggenenti P. Clinical practice. Nephropathy in patients with type 2 diabetes. N Engl J Med. 2002;346(15):1145-51.

5. Callaghan BC, Cheng HT, Stables CL, Smith AL, Feldman EL. Diabetic neuropathy: clinical manifestations and current treatments. Lancet Neurol. 2012;11(6):521-34.

6. Peltier A, Goutman SA, Callaghan BC. Painful diabetic neuropathy. BMJ. 2014;6(348):Article ID g1799. https://doi.org/10.1136/bmj.g1799

7. Brown JJ, Pribesh SL, Baskette KG, Vinik AI. A comparison of screening tools for the early detection of peripheral neuropathy in adults with and without type 2 diabetes. J Diabetes Res. 2017;2017:1467213.

8. Currie CJ, Poole CD, Woehl A, et al. The financial costs of healthcare treatment for people with type 1 or type 2 diabetes in the UK with particular reference to differing severity of peripheral neuropathy. Diabet Med. 2007;24(2):187-94.

9. Perkins BA, Bril V. Diabetic neuropathy: a review emphasizing diagnostic methods. Clin Neurophysiol. $2003 ; 114(7): 1167-75$.

10. England JD, Gronseth GS, Franklin G, et al. Distal symmetrical polyneuropathy: definition for clinical research. Muscle Nerve. 2005;31(1):113-23.
11. Weisman A, Bril V, Ngo M, et al. Identification and prediction of diabetic sensorimotor polyneuropathy using individual and simple combinations of nerve conduction study parameters. PLoS One. 2013;8(3):e58783. https://doi.org/10.1371/journal. pone.0058783

12. Wu CZ, Chang LC, Lin YF, et al. Urokinase plasminogen activator receptor and its soluble form in common biopsy-proven kidney diseases and in staging of diabetic nephropathy. Clin Biochem. 2015;48(18):1324-9.

13. Lin $\mathrm{X}, \mathrm{Xu} \mathrm{L}$, Zhao D, Luo Z, Pan S. Correlation between serum uric acid and diabetic peripheral neuropathy in T2DM patients. J Neurol Sci. 2018;385:78-82.

14. Olaleye D, Perkins BA, Bril V. Evaluation of three screening tests and a risk assessment model for diagnosing peripheral neuropathy in the diabetes clinic. Diabetes Res Clin Pract. 2001;54(2):115-28.

15. Zhu X, Mao F, Liu S, Zheng H, Lu B, Li Y. Association of SUDOSCAN values with vibration perception threshold in Chinese patients with type 2 diabetes mellitus. Int J Endocrinol. 2017; Article ID 8435252. https://doi.org/10.1155/2017/8435252

16. Williams $\mathrm{KH}$, Burns $\mathrm{K}$, Constantino $\mathrm{M}$, et al. An association of large-fibre peripheral nerve dysfunction with non-invasive measures of liver fibrosis secondary to non-alcoholic fatty liver disease in diabetes. J Diabetes Complicat. 2015;29(8):1240-7.

17. Shehab DK, Al-Jarallah KF, Abraham M, Mojiminiyi OA, Al-Mohamedy H, Abdella NA. Back to basics: ankle reflex in the evaluation of peripheral neuropathy in type 2 diabetes mellitus. QJM. 2012;105(4):315-20.

18. Tesfaye S, Boulton AJ, Dyck PJ, et al. Diabetic neuropathies: update on definitions, diagnostic criteria, estimation of severity, and treatments. Diabetes Care. 2010;33(10):2285-93.

19. Kamei N, Yamane K, Nakanishi S, et al. Effectiveness of Semmes-Weinstein monofilament examination for diabetic peripheral neuropathy screening. J Diabetes Complicat. 2005;19(1):47-53.

20. Rahman M, Griffin SJ, Rathmann W, Wareham NJ. How should peripheral neuropathy be assessed in people with diabetes in primary care? A populationbased comparison of four measures. Diabet Med. 2003;20(5):368-74.

21. Kim ES, Lee SW, Mo EY, Moon SD, Han JH. Inverse association between serum total bilirubin levels and diabetic peripheral neuropathy in patients with type 2 diabetes. Endocrine. 2015;50(2):405-12. 
22. Perkins BA, Orszag A, Ngo M, Ng E, New P, Bril V. Prediction of incident diabetic neuropathy using the monofilament examination: a 4-year prospective study. Diabetes Care. 2010;33(7):1549-54.

23. Martin CL, Waberski BH, Pop-Busui R, et al. Vibration perception threshold as a measure of distal symmetrical peripheral neuropathy in type 1 diabetes: results from the DCCT/EDIC study. Diabetes Care. 2010;33(12):2635-41.

24. Thomas GN, Lin JW, Lam WW, et al. Albuminuria is a marker of increasing intracranial and extracranial vascular involvement in Type 2 diabetic Chinese patients. Diabetologia. 2004;47(9):1528-34.

25. Chan JC, Cheung CK, Cheung MY, Swaminathan R, Critchley JA, Cockram CS. Abnormal albuminuria as a predictor of mortality and renal impairment in Chinese patients with NIDDM. Diabetes Care. 1995;18(7):1013-6.

26. Kotlarsky P, Bolotin A, Dorfman K, Knyazer B, Lifshitz T, Levy J. Link between retinopathy and nephropathy caused by complications of diabetes mellitus type 2. Int Ophthalmol. 2015;35(1):59-66.

27. Beilin J, Stanton KG, McCann VJ, Knuiman MW, Divitini ML. Microalbuminuria in type 2 diabetes: an independent predictor of cardiovascular mortality. Aust N Z J Med. 1996;26(4):519-25.
28. Bruno G, Merletti F, Bargero G, et al. Estimated glomerular filtration rate, albuminuria and mortality in type 2 diabetes: the Casale Monferrato study. Diabetologia. 2007;50(5):941-8.

29. Savage S, Estacio RO, Jeffers B, Schrier RW. Urinary albumin excretion as a predictor of diabetic retinopathy, neuropathy, and cardiovascular disease in NIDDM. Diabetes Care. 1996;19(11):1243-8.

30. $\mathrm{Hu} \mathrm{Y,} \mathrm{Liu} \mathrm{F,} \mathrm{Shen} \mathrm{J,} \mathrm{et} \mathrm{al.} \mathrm{Association} \mathrm{between}$ serum cystatin $\mathrm{C}$ and diabetic peripheral neuropathy: a cross-sectional study of a Chinese type 2 diabetic population. Eur J Endocrinol. 2014;171(5):641-8.

31. Callaghan BC, Price RS, Feldman EL. Distal symmetric polyneuropathy: a review. JAMA. 2015;314(20):2172-81.

32. Chuang YC, Chung MS, Wang PW, et al. Albuminuria is an independent risk factor of erectile dysfunction in men with type 2 diabetes. J Sex Med. 2012;9(4):1055-64.

33. Zhang Y, Jiang Y, Shen X, Yan S. Can both normal and mildly abnormal albuminuria and glomerular filtration rate be a danger signal for diabetic peripheral neuropathy in type 2 diabetes mellitus? Neurol Sci. 2017;38:1381-90. 